\title{
Knockdown of IncRNA HNF1A-AS1 inhibits oncogenic phenotypes in colorectal carcinoma
}

\author{
WENYI ZHU ${ }^{1,2^{*}}$, PEIPEI ZHUANG ${ }^{3 *}$, WEN SONG $^{1,2}$, SHIYU DUAN $^{1,2}$, \\ QIONG XU ${ }^{1}$, MAN PENG $^{1}$ and JUN ZHOU ${ }^{1,2}$ \\ ${ }^{1}$ Department of Pathology, Nanfang Hospital; ${ }^{2}$ Department of Pathology, School of Basic Medical Sciences; \\ ${ }^{3}$ Guangdong Provincial Key Laboratory of Molecular Tumor Pathology, Southern Medical University, \\ Guangzhou, Guangdong 510515, P.R. China
}

Received September 7, 2016; Accepted May 24, 2017

DOI: $10.3892 / \mathrm{mmr} .2017 .7175$

\begin{abstract}
Long non-coding RNAs (lncRNAs) have been demonstrated to serve important roles in the development and progression of cancer. Recently HNF1A antisense RNA 1 (HNF1A-AS1), a lncRNA, has been reported as exhibiting a potential oncogenic role in the development of many types of cancer. However, the expression and the role of HNF1A-AS1 in colorectal carcinoma (CRC) remains unclear. In the present study, the role of HNF1A-AS1 in CRC was examined for the first time and its correlation with CRC cell biological behaviors was analyzed. The results demonstrated that HNF1A-AS1 was distinctly upregulated in CRC tissues and associated with CRC metastasis to the lymph nodes. Reverse transcription-quantitative polymerase chain reaction revealed that HNF1A-AS1 was also upregulated in CRC cell lines and localized in the nucleus. In addition, knockdown of HNF1A-AS1 expression notably inhibited CRC cell proliferation, migration, invasion and colony formation, and suppressed S-phase entry in vitro. Taken together, these results suggested that HNF1A-AS1 might serve as a promising prognostic marker for CRC tumorigenesis and progression.
\end{abstract}

\section{Introduction}

Colorectal carcinoma (CRC) is one of the most common cancers worldwide. It is one of the five most commonly diagnosed cancers and leading causes of cancer death in China (1). Despite its common occurrence, the underlying mechanisms

Correspondence to: Professor Jun Zhou, Department of Pathology, School of Basic Medical Sciences, Southern Medical University, 1838 Guangzhou North Road, Guangzhou, Guangdong 510515, P.R. China

E-mail: chuhang127@163.com

*Contributed equally

Key words: HNF1A antisense RNA 1, colorectal cancer, long non-coding RNA, metastasis, proliferation of CRC pathogenesis remain poorly understood (2). Thus, discovering molecular markers and studying their functions in CRC may be of help to understand this neoplasm and to develop novel targets for targeted therapeutic interventions.

Long noncoding RNAs (lncRNAs) are a class of transcribed RNA molecules $>200$ nucleotides with no proteincoding capacity (3). In the past few years, studies have confirmed that lncRNAs are indispensable in many biological events, including cell differentiation, cell cycle and apoptosis, through comprehensive mechanisms (4). LncRNAs have been discovered to serve important roles in the genesis and progression of various types of cancer, acting as either oncogenes or tumor suppressors $(2,5)$. For example, the lncRNA HOXA cluster antisense RNA 2 promotes gastric cancer cell proliferation and tumorigenesis via epigenetically silencing cyclin-dependent kinase inhibitor 1A, polo-like kinase 3 and DNA damage-inducible transcript 3 transcription by binding with enhancer of zeste 2 polycomb repressive complex 2 subunit (6). Another lncRNA, FEZF1 antisense RNA 1, was confirmed to participate in colorectal tumorigenesis and progression through Fez family zinc finger protein 1 induction (7). Nonetheless, a large number of lncRNAs are probably still undiscovered and their functions in various biological events, including tumorigenesis, still remain unclear.

The lncRNA HNF1A antisense RNA 1 (HNF1A-AS1) is located on chromosome 12 and is transcribed as a 2,455 bp lncRNA. Recently, a next-generation sequencing analysis identified HNF1A-AS1 as a non-coding oncogene involved in the tumorigenesis of esophageal adenocarcinoma (8). Wu et al (9) reported that increased HNF1A-AS1 expression promotes cell proliferation and metastasis and identified HNF1A-AS1 as a poor prognostic biomarker in lung adenocarcinoma. In addition, previous studies have demonstrated that HNF1A-AS1 functions as an oncogene and a promoter of autophagy in hepatocellular carcinoma (HCC), through repressing hsa-miR-30b-5p and activating BCL2 apoptosis regulator (10). These results suggest that dysregulation of HNF1A-AS1 may participate in tumorigenesis or tumor progression. However, the functional role and underlying mechanism of HNF1A-AS1 in CRCs remain unclear.

In the present study, the effects of HNF1A-AS1 expression were examined on CRC. The results indicated that 
HNF1A-AS1 expression was upregulated in human CRC tissues and associated with lymph node metastasis. Further functional experiments revealed that HNF1A-AS1 may be important for increased cell proliferation, migration and invasiveness. Taken together, the present data indicated that HNF1A-AS1 may participate as a non-coding oncogene in $\mathrm{CRC}$ tumorigenesis.

\section{Materials and methods}

Cell culture. The human CRC cell lines SW480, SW620, DLD-1, HT29, HCT116, Ls-174T and LOVO were obtained from the Cell Bank of Type Culture Collection of the Chinese Academy of Sciences (Shanghai, China). All CRC cell lines were cultured in RPMI 1640 medium (Gibco; Thermo Fisher Scientific, Inc., Waltham, MA, USA) with $10 \%$ fetal bovine serum (FBS; HyClone; GE Healthcare Life Sciences, Logan, $\mathrm{UT}, \mathrm{USA}$ ) at $37^{\circ} \mathrm{C}$ in a humidified incubator containing $5 \% \mathrm{CO}_{2}$.

Clinical samples. Fresh colorectal tumor tissue samples and adjacent non-tumor tissues were obtained from patients who were diagnosed with primary CRC from January to June in 2010. Elective surgery was carried out on these patients at Nanfang Hospital, Southern Medical University (Guangzhou, China). In total, 14 cases of fresh CRC tissue and adjacent non-tumor tissues were freshly frozen in liquid nitrogen and stored at $-80^{\circ} \mathrm{C}$ until further use. The mean age of the patients was $60.1 \pm 12.4$ years; 5 of the 14 patients $(35.7 \%)$ were female and the remaining 9 were male (64.3\%). According to the tumor size, lymph node and metastasis classification system (11), among the patients the following was observed: 2 patients $(14.3 \%)$ were $\mathrm{T}$ stage 2 (T2), 9 patients $(64.3 \%)$ were T3 and 3 patients $(21.4 \%)$ were T4; 8 patients $(57.1 \%)$ had lymph-node metastasis and 6 patients $(42.9 \%)$ did not; 2 patients $(14.3 \%)$ developed a distant metastasis while the remaining patients did not. The use of tissues for this study has been approved by the Ethics Committee of Nanfang Hospital, Southern Medical University. Prior to using these clinical materials for research purposes, all the patients signed an informed consent.

RNA extraction and reverse transcription-quantitative polymerase chain reaction $(R T-q P C R)$. Total RNA was extracted using TRIzol reagent (Invitrogen; Thermo Fisher Scientific, Inc.). cDNA was synthesized using the PrimeScript RT reagent kit (Takara Biotechnology Co., Ltd., Dalian, China). qPCR was performed with the SYBR Premix Ex Taq kit (Takara Biotechnology Co., Ltd.) on an ABI 7500 Real-Time PCR system (Applied Biosystems; Thermo Fisher Scientific, Inc.). 3-step qPCR was performed with the following thermocycling conditions: $95^{\circ} \mathrm{C}$ for $10 \mathrm{~min}$, followed by 40 cycles of amplification $\left(95^{\circ} \mathrm{C}\right.$ for $5 \mathrm{sec}, 60^{\circ} \mathrm{C}$ for $20 \mathrm{sec}$ and $72^{\circ} \mathrm{C}$ for $34 \mathrm{sec})$ and then a dissociation stage profile $\left(95^{\circ} \mathrm{C}\right.$ for $15 \mathrm{sec}$, $60^{\circ} \mathrm{C}$ for $1 \mathrm{~min}$ and $95^{\circ} \mathrm{C}$ for $15 \mathrm{sec}$ ). GAPDH and U6 were used as an endogenous controls. Fold changes were calculated through the relative quantification $2^{-\Delta \Delta C q}$ method (12). Primer sequences are listed in Table I. To account for technical variability, the assay was performed in triplicate for each sample.

Subcellular fractionation. Nuclear and cytosolic RNA isolation was performed as described previously (13), with some
Table I. Primer sequences used for reverse transcriptionquantitative polymerase chain reaction.

\begin{tabular}{lll}
\hline Gene & Primer & \multicolumn{1}{c}{ Sequence $\left(5^{\prime}-3^{\prime}\right)$} \\
\hline HNF1A-AS1 & Forward & TCAAGAAATGGTGGCTAT \\
& Reverse & GATCTGAGACTGGCTGAA \\
GAPDH & Forward & ACAGTCAGCCGCATCTTCTT \\
& Reverse & GACAAGCTTCCCGTTCTCAG \\
U6 & Forward & CTCGCTTCGGCAGCACA \\
& Reverse & AACGCTTCACGAATTTGCGT \\
\hline
\end{tabular}

HNF1A-AS1, HNF1A antisense RNA 1; U6, U6 small nuclear RNA.

modifications. Briefly, cells were rinsed twice with ice-cold PBS centrifuged at $111.8 \mathrm{x} \mathrm{g}$ at $4^{\circ} \mathrm{C}$ for $5 \mathrm{~min}$. Cell pellets were resuspended by gentle pipetting in $150 \mu 10.1 \% \mathrm{NP}-40$ (dissolved in water), and incubated on ice for $5 \mathrm{~min}$, followed by centrifugation at $5,000 \mathrm{xg}$ at $4^{\circ} \mathrm{C}$ for $1 \mathrm{~min}$. The resulting lysate was the cytosolic fraction of cells, which was added in TRIzol reagent for cytosolic RNA extraction. Following gentle rinsing and centrifugation at $111.8 \mathrm{x} \mathrm{g}$ at $4^{\circ} \mathrm{C}$ for $5 \mathrm{~min}$ twice, the cell pellets were resuspended by gentle pipetting in $75 \mu \mathrm{l}$ $0.1 \% \mathrm{NP}-40$ and incubated on ice for $5 \mathrm{~min}$. TRIzol reagent was then added to the lysate for nuclear RNA purification.

Transfection of CRC cells. HCT116 and HT29 cells were seeded into six-well plates. The sequence of the small interfering RNA (siRNA) to knockdown HNF1A-AS1 was: 5'-CAC CUGCAUUCAAACUCGGACUGUU-3'. The target sequence of siRNA-HNF1A-AS1 was: 5'-CACCTGCATTCAAACTCG GACTGTT-3'. The sequence of the negative control siRNA was: 5'-UUGUACUACACAAAAGUACUG-3'. HNF1A-AS1 siRNA and negative control siRNA were transfected (0.1 nmol siRNA/well) into HCT116 and HT29 cells using Lipofectamine 3000 (Invitrogen; Thermo Fisher Scientific, Inc.) according to the manufacturer's instructions. Cells were harvested $48 \mathrm{~h}$ post-transfection for RT-qPCR and functional experiments.

Cell proliferation assay. Cell Counting Kit-8 (CCK-8) assay (Dojindo Molecular Technologies, Inc., Rockville, MD, USA) was used in order to evaluate the rate of cell proliferation, according to manufacturer's instructions. Briefly, log-phase cells were trypsinized into a single-cell suspension and plated into 96-well plates at a density of 1,000 cells/well. CCK-8 solution was added to each well. After $2 \mathrm{~h}$, the absorbance of each well was measured at $450 \mathrm{~nm}$ using a microplate reader (Enspire 2300 Multimode plate reader; PerkinElmer, Inc., Waltham, MA, USA).

Colony formation assay. Experiments were performed based on those previously described (14). The cells were plated in 6-well plates at $2 \times 10^{2}$ cells/well and maintained in RPMI 1640 containing 10\% FBS for 2 weeks. After 2 weeks, the cells were washed twice with PBS, fixed with methanol and stained with Giemsa. The number of colonies with diameter $>150 \mu \mathrm{m}$ was counted using Image Pro Plus v6.0 (Media Cybernetics, 
Inc., Rockville, MD, USA); whole plates were counted and 3 replicate plates were performed per sample. A total of 3 independent experiments were performed.

Cell migration assay. Cell motility was measured using a wound healing assay by measuring the movement of cells in a scraped, acellular area created by a $10 \mu \mathrm{l}$ pipette tube. Wound closure was observed at 0 and $48 \mathrm{~h}$. Photographs were captured in order to assess the level of migration in each group of transfected cells. The migration was quantified by counting the total number of cells that migrated toward the original wound field. A total of 5 random fields were measured per plate, 3 plates per sample, and 3 independent experiments were performed using Image Pro Plus v6.0 (Media Cybernetics Corporation, USA) for analysis.

Cell invasion assay. For invasion assay, Matrigel-coated chambers (BD Biosciences, San Jose, CA, USA) containing $8-\mu \mathrm{m}$ pores were used. Cells were seeded in the upper chambers (coated in Matrigel) at $1 \times 10^{5}$ concentration in serum-free medium. The lower chamber of the transwells was filled with culture media containing $10 \%$ FBS as a chemoattractant. After incubation at $37^{\circ} \mathrm{C}$ for $48 \mathrm{~h}$, non-invaded cells on the top of the transwells were scraped off with a cotton swab. The successfully invaded cells at the bottom of the transwells were fixed with methanol and stained with Giemsa for $15 \mathrm{~min}$, prior to counting under a light microscope. A total of 5 random fields were measured per plate, 3 plates per samples, and 3 independent experiments were performed.

Flow cytometric analysis. Cells were seeded at a density of $1 \times 10^{6}$ cells/well in six-well plates. After $48 \mathrm{~h}$, cells were washed with PBS and then treated with a Cell Cycle Staining kit [MultiSciences (Lianke) Biotech Co., Ltd., Hangzhou, China] for $30 \mathrm{~min}$ at $4^{\circ} \mathrm{C}$ in the dark. The cell cycle profiles were analyzed using a Elite ESP flow cytometer (Beckman Coulter, Inc., Brea, CA, USA) at $488 \mathrm{~nm}$, and data were analyzed with the CellQuest software v3.1 (BD Biosciences).

Statistical analysis. All statistical analyses were performed using the SPSS v16.0 statistical software package (SPSS, Inc., Chicago, IL, USA). Data were presented as mean \pm standard error of the mean from at least three independent experiments. The differences between variables were assessed using the following statistical tests: t-test, factorial analysis, $\chi^{2}$-test, Fisher's exact test, or one-way analysis of variance. Multiple comparisons between groups were performed using the Student-Newman-Keuls post hoc test. $\mathrm{P}<0.05$ was considered to indicate a statistically significant difference.

\section{Results}

LncRNA HNF1A-AS1 is upregulated in CRC tissues and associated with metastasis. As a first step towards understanding the role of lncRNA HNF1A-AS1 in CRC, the expression of HNF1A-AS1 was examined in CRC tissues. RT-qPCR was performed to detect the expression of HNF1A-AS1 in a total of 14 paired clinical CRC tissues and adjacent non-tumor tissues. The results revealed that HNF1A-AS1 expression levels in tumors were significantly higher compared with the paired normal tissues (Fig. 1A). Next, the association between HNF1A-AS1 expression and the lymph-node metastasis status of the CRC samples was examined. The results revealed that upregulation of HNF1A-AS1 was significantly associated with lymph-node metastasis of CRC (Fig. 1B). Taken together, these results suggested that HNF1A-AS1 might have an important role in CRC pathogenesis and progression.

HNF1A-AS1 is upregulated in CRC cell lines and localized in the nucleus. Since HNF1A-AS1 was highly expressed in CRC tissues, the role of HNF1A-AS1 was further examined in CRC cell lines. RT-qPCR was used to examine the expression levels of HNF1A-AS1 in seven CRC cell lines (SW480, SW620, DLD-1, HT29, HCT116, Ls-174T and LOVO). The results demonstrated that the HCT116 and HT29 cell lines displayed the highest expression levels of HNF1A-AS1 (Fig. 1C). In order to determine the subcellular localization of HNF1A-AS1, a nuclear/cytosolic fractionation was performed on HT116 and HT29 cells, followed by RT-qPCR. The differential enrichment of GAPDH and U6 RNAs were used as fractionation indicators for the cytosolic and nuclear extracts respectively. The results revealed markedly higher levels of HNF1A-AS1 in the nucleus compared with the cytosol in both cell lines tested (Fig. 1D), which indicated that HNF1A-AS1 was mainly localized in the nuclear fraction of CRC cells.

Knockdown of HNF1A-AS1 inhibits CRC cell proliferation and colony formation in vitro. To further investigate the impact of HNF1A-AS1 on CRC cell biological behaviors, specific siRNA was employed to knockdown HNF1A-AS1 expression in CRC cells. According to endogenous HNF1A-AS1 expression in the CRC cell lines mentioned above, the HCT116 and HT29 cell lines were selected for HNF1A-AS1 knockdown by RNA interference. RT-qPCR analysis revealed that HNF1A-AS1 expression was downregulated by $62.13 \%$ in HCT116 cells and by $52.18 \%$ in HT29 cells following specific siRNA transfection compared with negative control siRNA transfection (Fig. 1E). Growth curves determined by CCK-8 assay indicated that cell proliferation was reduced following HNF1A-AS1 knockdown in HCT116 and HT29 cells (Fig. 2A and B). Notably, the results from the colony formation assay also demonstrated that decreased HNF1A-AS1 expression resulted in a decreased colony formation ability in HCT116 and HT29 cells (Fig. 2C).

HNF1A-AS1 promotes CRC cell migration and invasion in vitro. The effect of HNF1A-AS1 on cell migration and invasion was next assessed in vitro. The migration ability of HCT116 and HT29 cells was determined by wound healing assay. The results demonstrated that knockdown of HNF1A-AS1 significantly inhibited HCT116 and HT29 cell migration compared with the negative control cells (Fig. 2D). Furthermore, Matrigel invasion assay revealed that knockdown of HNF1A-AS1 inhibited the invasion ability of HCT116 cells by $74.62 \%$ and of HT29 cells by $57.23 \%$ compared with the negative control cells (Fig. 2E). These results indicated that the downregulation of HNF1A-AS1 expression effectively reduced both migration and invasion of CRC cells in vitro. 
A

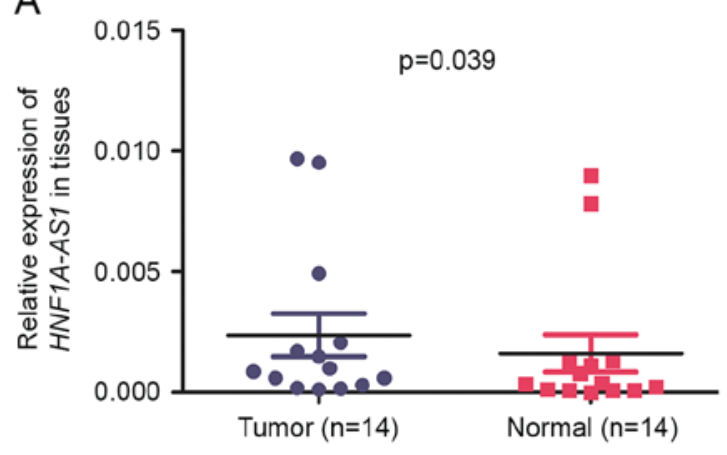

C

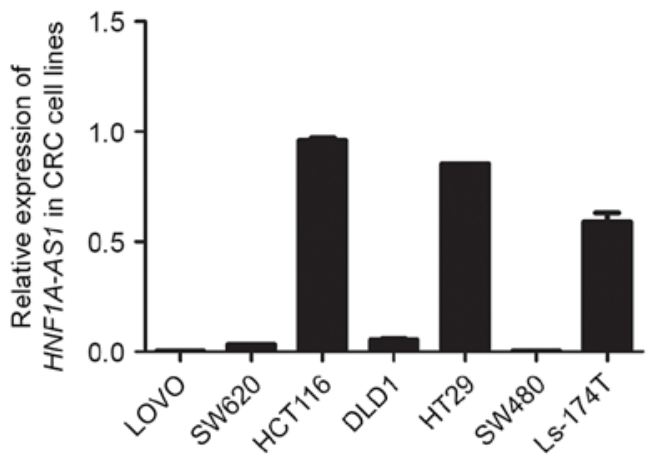

B
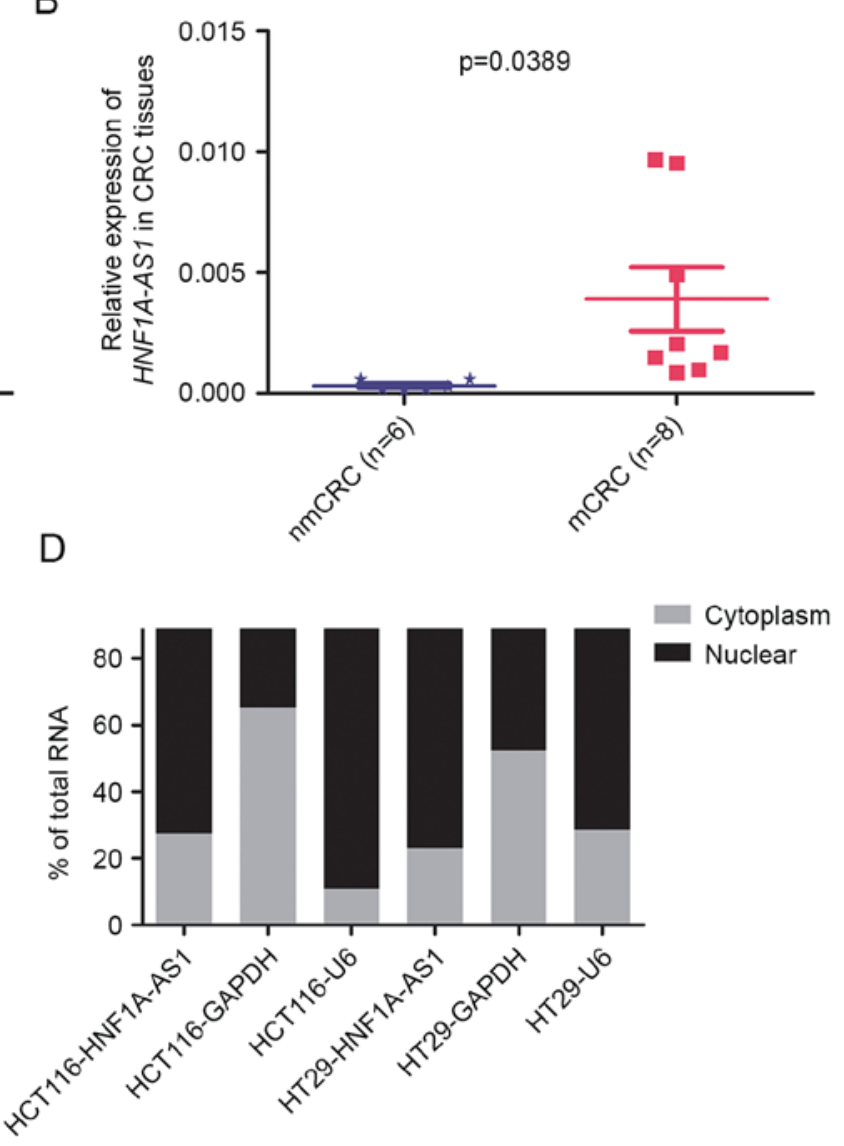

$\mathrm{E}$

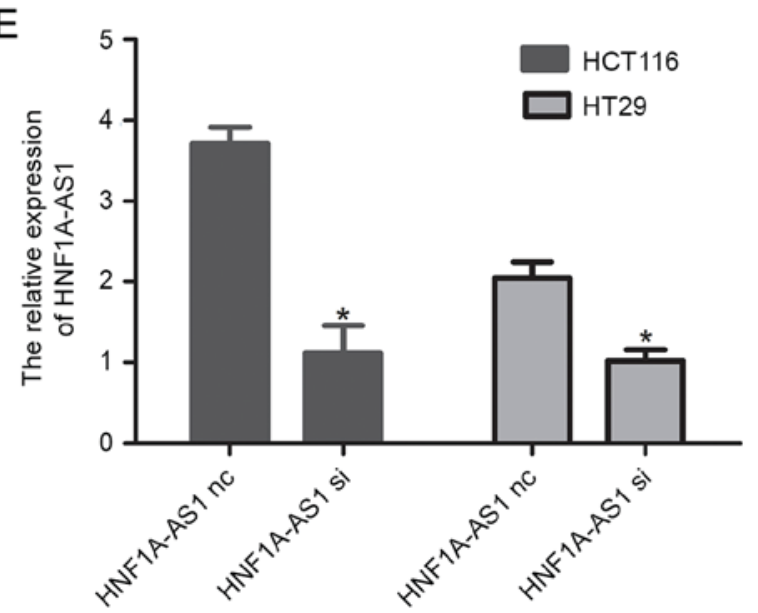

Figure 1. Analysis of HNF1A-AS1 expression in CRC tissues and cells. (A) Relative expression of HNF1A-AS1 in paired CRC tissues and adjacent non-tumor tissues ( $\mathrm{n}=14)$. HNF1A-AS1 expression was examined by RT-qPCR and normalized to GAPDH expression. (B) The HNF1A-AS1 expression in CRC tissues with (mCRC) or without (nmCRC) lymph-node metastasis. (C) Relative expression of HNF1A-AS1 in seven CRC cell lines. HNF1A-AS1 expression was examined by RT-qPCR and normalized to GAPDH expression. (D) HNF1A-AS1 cell localization, as identified using RT-qPCR in fractionated HCT116 and HT29 cells. GAPDH and U6 were used as cytosolic and nuclear fractionation indicators, respectively. (E) Relative expression of HNF1A-AS1 was measured in HCT116 and HT29 cells by RT-qPCR following transfection with either a specific siRNA (si) or a negative control siRNA (nc). * $<0.05$ vs. nc group. HNF1A-AS1, HNF1A antisense RNA 1; CRC, colorectal carcinoma; RT-qPCR, reverse transcription-quantitative polymerase chain reaction; m, metastatic; nm, non-metastatic; U6, U6 small nuclear RNA; siRNA, small interfering RNA; nc, negative control.

Knockdown of HNF1A-AS1 promotes G1 arrest. To further examine the role of HNF1A-AS1 in CRC, the relationship between HNF1A-AS1 knockdown and cell cycle progression was assessed. Cell cycle phase distribution was evaluated in HCT116 and HT29 cells by flow cytometry. The results demonstrated that knockdown of HNF1A-AS1 resulted in a significant increase of the $\%$ of cells at G0/G1 phase, but a significant decrease in the $\%$ of cells in the $\mathrm{S}$ phase of the cell cycle (Fig. 3).

\section{Discussion}

Colorectal carcinoma (CRC) has a high occurrence worldwide, however, the mechanisms underlying its pathogenesis 
A

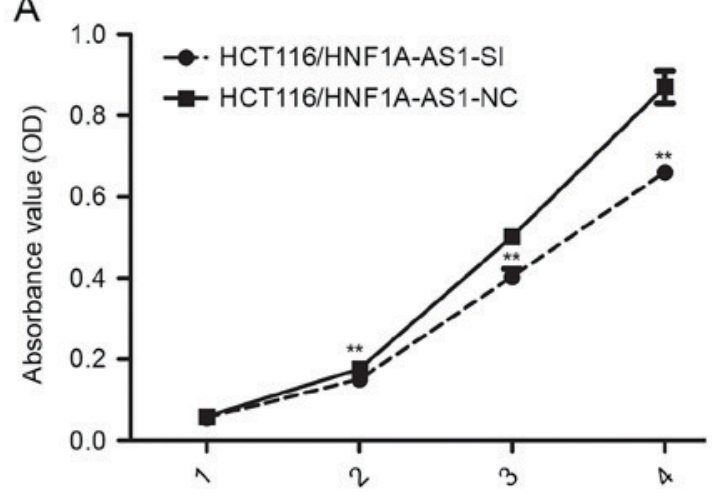

B

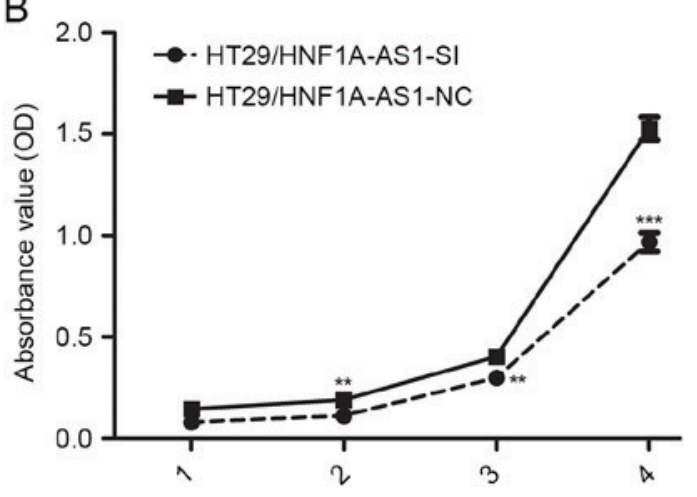

C

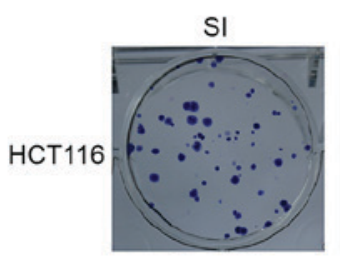

NC
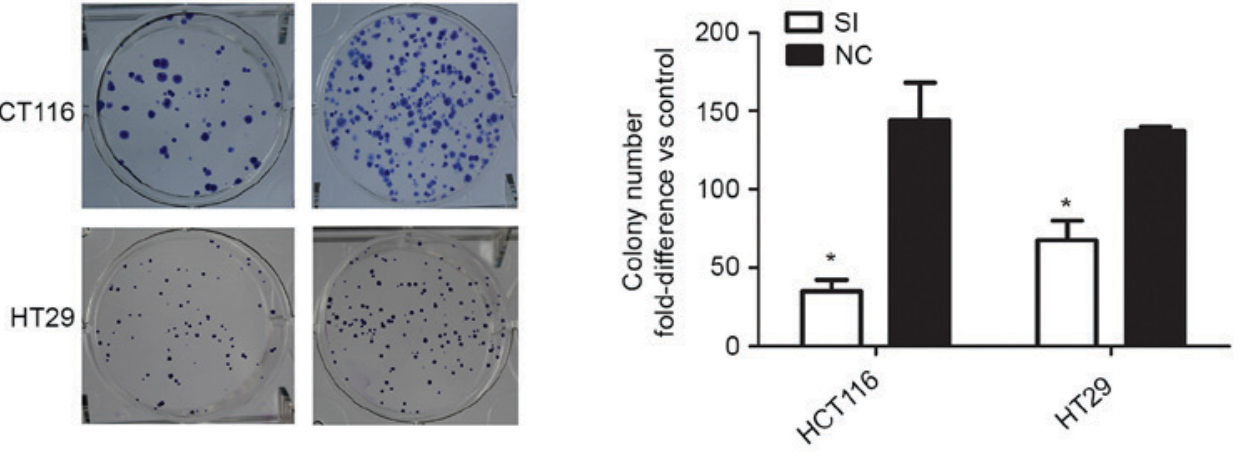

D
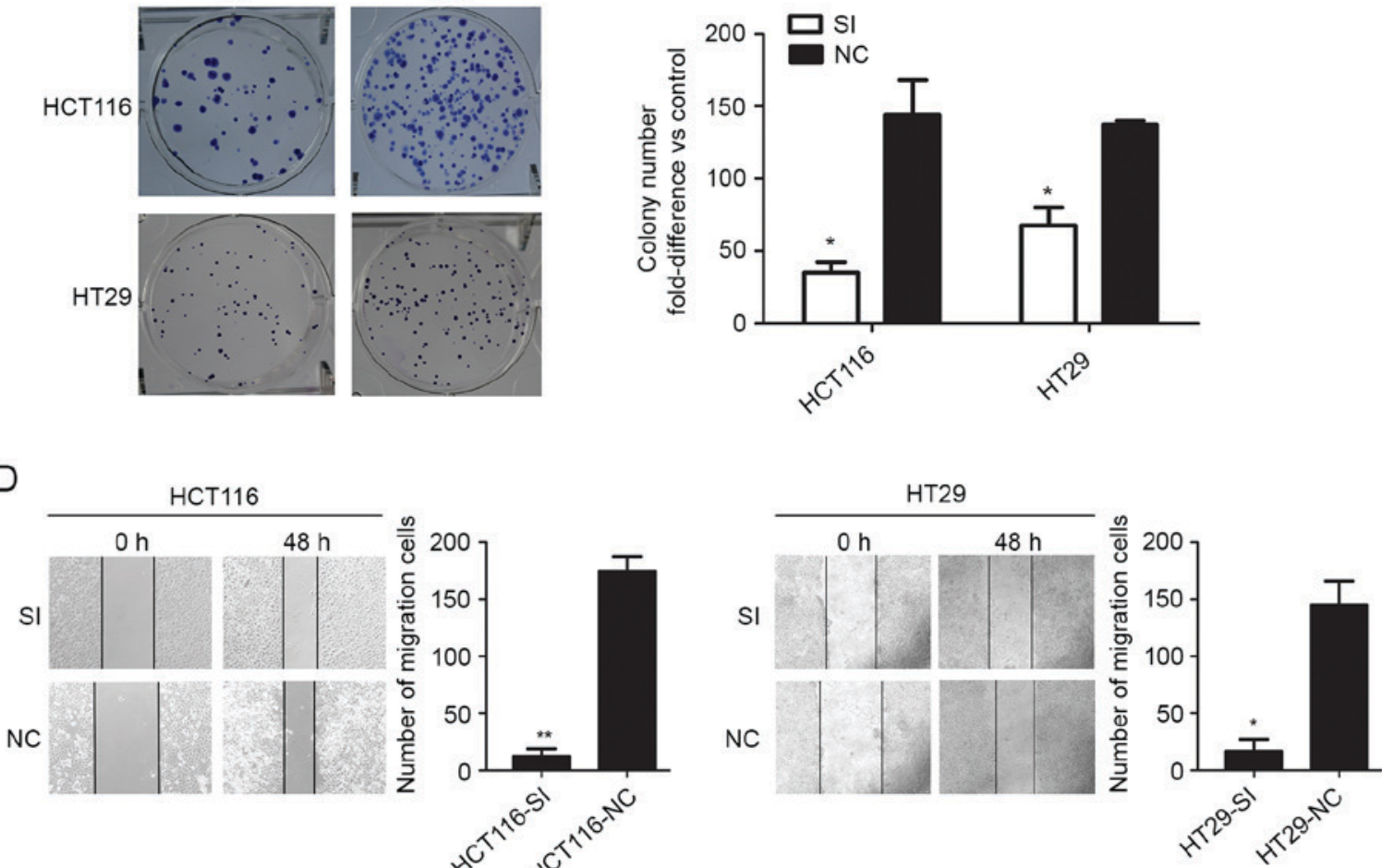

E
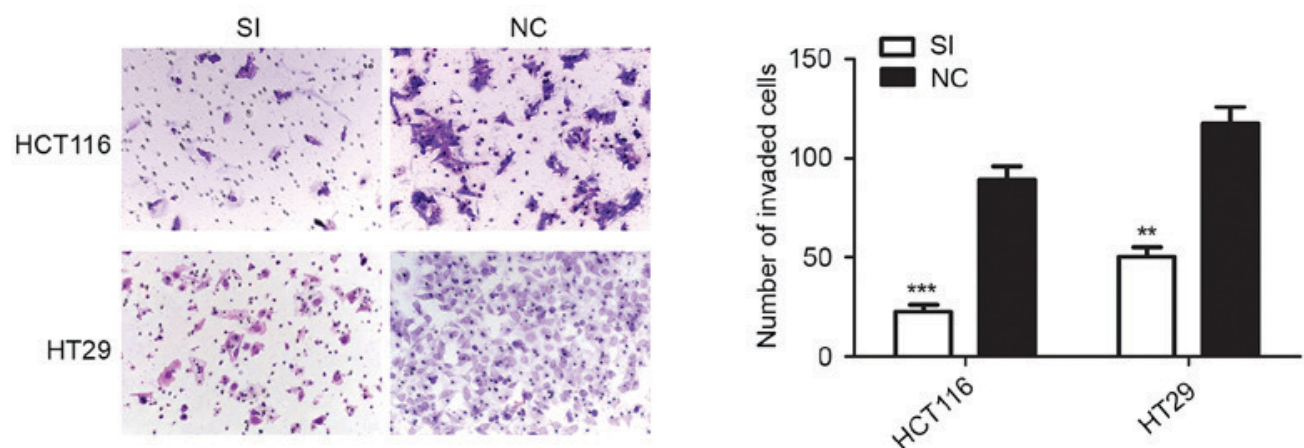

Figure 2. HNF1A-AS1 knockdown inhibits CRC cell migration, invasion, proliferation and colony formation in vitro. HCT116 and HT29 cells were transfected with either a HNF1A-AS1 specific siRNA or a negative control siRNA. (A) Cell proliferation was evaluated by CCK-8 assay in HCT116 cells. (B) Cell proliferation was evaluated by CCK-8 assay in HT29 cells. (C) Colony-forming assays were performed to determine the growth of CRC cells. (D) Migration ability was tested by a scratch-wound healing assay (magnification, x100). (E) Invasion ability was tested in matrigel-coated transwell invasion chambers (magnification, $\mathrm{x} 200$ ). ${ }^{*} \mathrm{P}<0.05,{ }^{* *} \mathrm{P}<0.01$ and ${ }^{* * * *} \mathrm{P}<0.001$ vs. NC. HNF1A-AS1, HNF1A antisense RNA 1; CRC, colorectal carcinoma; siRNA, small interfering RNA; CCK-8, Cell Counting Kit-8; SI, siRNA; NC, negative control.

are still unclear. LncRNAs are a recently discovered class of transcribed RNA molecules that have been implicated in the etiology and progression of cancer (15-18). Previous studies have identified HNF1A-AS1 as important in cell proliferation, cell cycle regulation, migration and invasion of human esophageal adenocarcinomas, lung adenocarcinomas and HCC (8-10). 
A

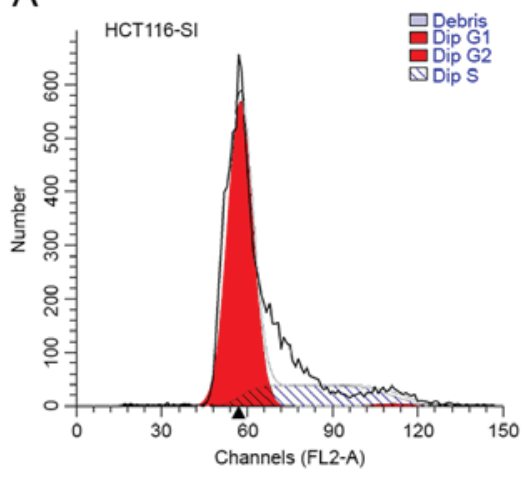

B

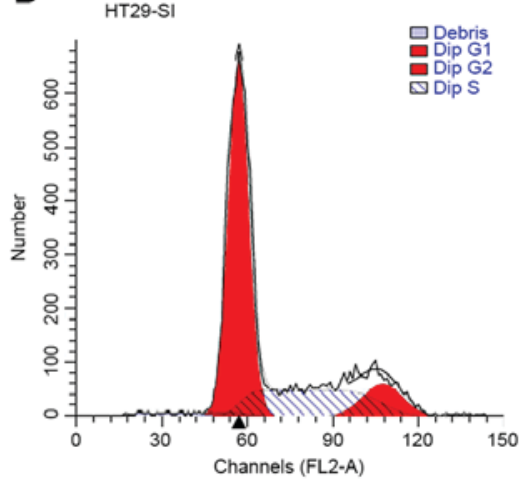

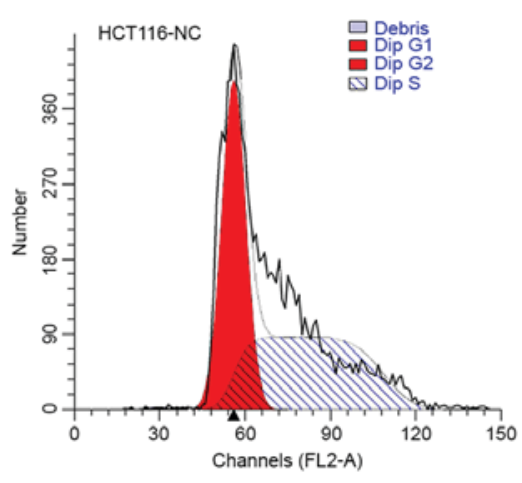
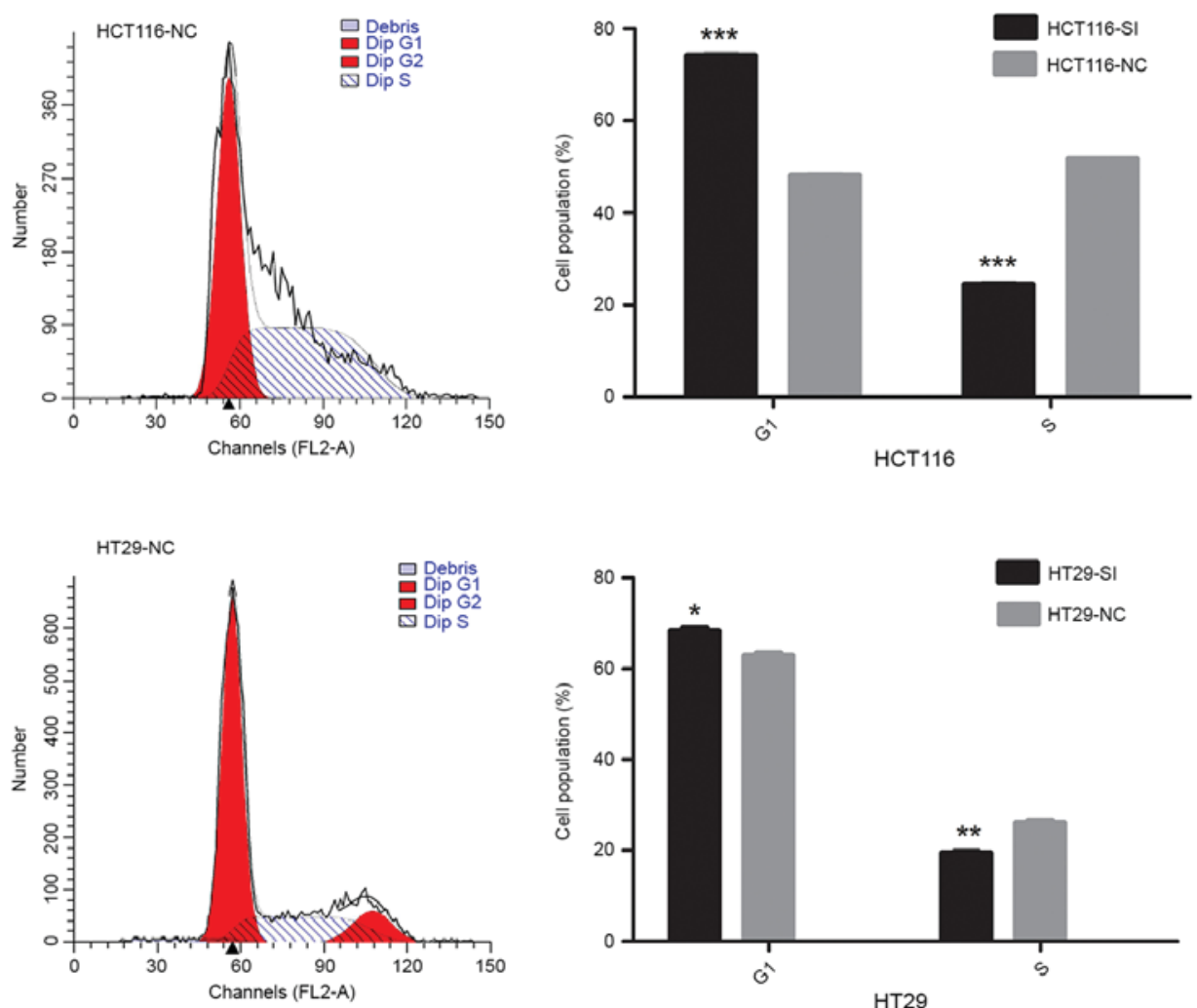

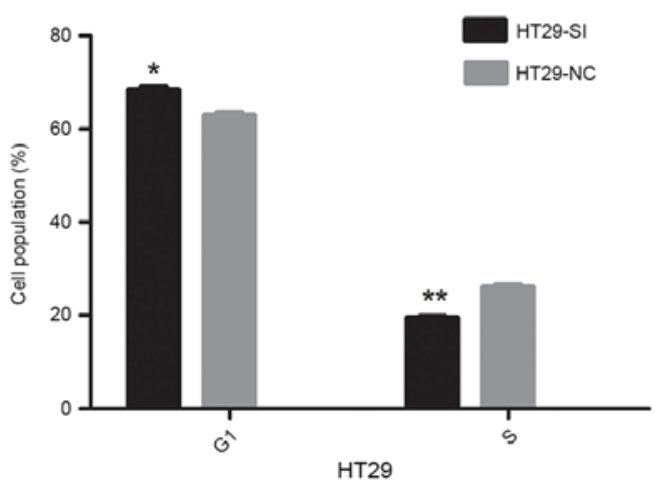

Figure 3. Effect of HNF1A-AS1 knockdown on CRC cell cycle progression in vitro. (A) HCT116 and (B) HT29 cells were transfected with either a HNF1A-AS1 specific siRNA or a negative control siRNA and cell cycle analysis was performed at 48 h post-transfection by flow cytometry. Data were presented as the mean \pm standard deviation from three independent experiments. ${ }^{*} \mathrm{P}<0.05,{ }^{* *} \mathrm{P}<0.01$ and ${ }^{* * *} \mathrm{P}<0.001$ vs. NC. HNF1A-AS1, HNF1A antisense RNA 1 ; CRC, colorectal carcinoma; siRNA, small interfering RNA; SI, siRNA; NC, negative control.

However, the potential role and associated molecular mechanisms of HNF1A-AS1 in CRC are yet to be clarified.

In order to explore the relationship of HNF1A-AS1 in $\mathrm{CRC}$, clinical tissues from patients with CRC were analyzed for HNF1A-AS1 expression by RT-qPCR. HNF1A-AS1 levels were significantly higher in tumors compared with paired normal tissues, and its high expression was also significantly associated with lymph node metastasis. The present study therefore suggests that HNF1A-AS1 might be important in the progression of CRC.

Next, the expression of HNF1A-AS1 was examined in seven CRC cell lines and it was discovered that HNF1A-AS1 localized in the nucleus. Specific siRNA was employed to knockdown HNF1A-AS1 expression in HCT116 and HT29 cells, in order to test its function in key tumor cell behaviors. The present results revealed that HNF1A-AS1 knockdown significantly inhibited CRC cell proliferation, migration and invasion, and suppressed S-phase entry in vitro. These findings indicate that HNF1A-AS1 may serve as an oncogene by promoting CRC development and progression.

The importance of lncRNAs in human cancer may be associated with their abilities to impact cellular functions through various mechanisms. Previous studies have shown that HNF1A-AS1 knockdown inhibited cell proliferation in vitro in human esophageal adenocarcinoma cells, lung adenocarcinoma cells and HCC models (8-10). In the present study, HNF1A-AS1 was also demonstrated to act as an oncogene in CRC. LncRNAs can function in cooperation with various molecules to promote tumorigenesis. Therefore, the next step for future studies exploring the role of HNF1A-AS1 in CRC will be to identify its potential interacting partners or targets. Based on previous studies, it is hypothesized that HNF1A-AS1 might contribute to CRC development and progression through regulating HNF1A. To this end, future studies should investigate the relationship of these two molecules and their expression in CRC tissues and cell lines, and loss-of-function experiments for HNF1A would be crucial in order to determine its functional role. In addition, lncRNA H19 has been reported to be significantly inhibited by HNF1A-AS1 knockdown in esophageal adenocarcinoma cells (8). HNF1A-AS1 also influences lung adenocarcinoma cell epithelial-mesenchymal transition by regulating cyclin D1, E-cadherin, N-cadherin and $\beta$-catenin expression (9). Furthermore, HNF1A-AS1 serves as an autophagy promoter in $\mathrm{HCC}$ through repressing miR-30b-5p (10). Therefore, all these molecules may be important functional targets of HNF1A-AS1 in CRC cells. Future studies will be required to fully elucidate the molecular mechanisms by which HNF1A-AS1 may promote pathogenesis and progression in CRC.

\section{Acknowledgements}

This study was supported by the National Nature Science Fund of China (grant nos. 81272763 and 81672466) and the Natural Science Foundation of Guangdong Province (grant no. 2014A030313286). 


\section{References}

1. Chen W, Zheng R, Baade PD, Zhang S, Zeng H, Bray F, Jemal A, Yu XQ and He J: Cancer statistics in China, 2015. CA Cancer J Clin 66: 115-132, 2016.

2. Han D, Wang M, Ma N, Xu Y, Jiang Y and Gao X: Long noncoding RNAs: Novel players in colorectal cancer. Cancer Lett 361: 13-21, 2015.

3. Tsai MC, Spitale RC and Chang HY: Long intergenic noncoding RNAs: New links in cancer progression. Cancer Res 71: 3-7, 2011.

4. Zhang QQ, Xu MY, Qu Y, Hu JJ, Li ZH, Zhang QD and Lu LG: TET3 mediates the activation of human hepatic stellate cells via modulating the expression of long non-coding RNA HIF1A-AS1. Int J Clin Exp Pathol 7: 7744-7751, 2014.

5. Smolle M, Uranitsch S, Gerger A, Pichler M and Haybaeck J: Current status of long non-coding RNAs in human cancer with specific focus on colorectal cancer. Int J Mol Sci 15: 13993-14013, 2014.

6. Han P, Li W, Lin CH, Yang J, Shang C, Nurnberg ST, Jin KK, $\mathrm{Xu} \mathrm{W}$, Lin CY, Lin CJ, et al: A long noncoding RNA protects the heart from pathological hypertrophy. Nature 514: 102-106, 2014.

7. Chen N, Guo D, Xu Q, Yang M, Wang D, Peng M, Ding Y, Wang S and Zhou J: Long non-coding RNA FEZF1-AS1 facilitates cell proliferation and migration in colorectal carcinoma. Oncotarget 7: 11271-11283, 2016.

8. Yang X, Song JH, Cheng Y, Wu W, Bhagat T, Yu Y, Abraham JM, Ibrahim S, Ravich W, Roland BC, et al: Long non-coding RNA HNF1A-AS1 regulates proliferation and migration in oesophageal adenocarcinoma cells. Gut 63: 881-890, 2014.

9. Wu Y, Liu H, Shi X, Yao Y, Yang W and Song Y: The long non-coding RNA HNF1A-AS1 regulates proliferation and metastasis in lung adenocarcinoma. Oncotarget 6: 9160-9172, 2015.

10. Liu Z, Wei X, Zhang A, Li C, Bai J and Dong J: Long non-coding RNA HNF1A-AS1 functioned as an oncogene and autophagy promoter in hepatocellular carcinoma through sponging hsa-miR-30b-5p. Biochem Bioph Res Commun 473: 1268-1275, 2016.
11. Markowitz SD and Bertagnolli MM: Molecular origins of cancer: Molecular basis of colorectal cancer. New Engl J Med 361: 2449-2460, 2009.

12. Zhu X, Liang L and Ding Y: Overexpression of FMNL2 is closely related to metastasis of colorectal cancer. Int J Colorectal Dis 23: 1041-1047, 2008

13. Chen LL, DeCerbo JN and Carmichael GG: Alu elementmediated gene silencing. Embo J 27: 1694-1705, 2008.

14. Wu W, Bhagat TD, Yang X, Song JH, Cheng Y, Agarwal R, Abraham JM, Ibrahim S, Bartenstein M, Hussain Z, et al: Hypomethylation of noncoding DNA regions and overexpression of the long noncoding RNA, AFAP1-AS1, in Barrett's esophagus and esophageal adenocarcinoma. Gastroenterology 144: 956-966, 2013.

15. Deng R, Liu B, Wang Y, Yan F, Hu S, Wang H, Wang T, Li B, Deng X, Xiang S, et al: High expression of the newly found long noncoding RNA Z38 promotes cell proliferation and oncogenic activity in breast cancer. J Cancer 7: 576-586, 2016.

16. Salameh A, Lee AK, Cardó-Vila M, Nunes DN, Efstathiou E, Staquicini FI, Dobroff AS, Marchiò S, Navone NM, Hosoya H, et al: PRUNE2 is a human prostate cancer suppressor regulated by the intronic long noncoding RNAPCA3. Proc Natl Acad Sci USA 112: 8403-8408, 2015.

17. Liu F, Yuan JH, Huang JF, Yang F, Wang TT, Ma JZ, Zhang L, Zhou CC, Wang F, Yu J, et al: Long noncoding RNA FTX inhibits hepatocellular carcinoma proliferation and metastasis by binding MCM2 and miR-374a. Oncogene 35: 5422-5434, 2016.

18. Fang Z, Xu C, Li Y, Cai X, Ren S, Liu H, Wang Y, Wang F, Chen R, Qu M, et al: A feed-forward regulatory loop between androgen receptor and PlncRNA-1 promotes prostate cancer progression. Cancer Lett 374: 62-74, 2016. 